\title{
Transparência nos ensaios clínicos nas Américas: é preciso coordenar as esferas reguladoras*
}

\author{
Trudo Lemmens ${ }^{1}$ e Carlos Herrera Vacaflor ${ }^{2}$
}

\author{
Várias iniciativas contribuíram para a transparência dos dados de saúde nas Américas. Porém, \\ Trudo Lemmens e Carlos Herrera Vacaflor argumentam que são necessários mais esforços \\ coordenados para garantir a confiabilidade da pesquisa em saúde.
}

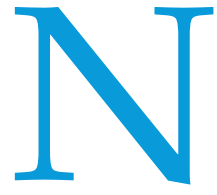
a sua Política de Pesquisa para a Saúde de 2009, a Organização PanAmericana da Saúde/ Organização Mundial da Saúde (OPAS/OMS) reiterou o papel fundamental da transparência para como fator garantidor de confiabilidade das pesquisas em saúde. ${ }^{1} \mathrm{Na}$ esteira de várias ocorrências altamente controversas, tem-se promovido cada vez mais a transparência, especialmente no contexto de pesquisas farmacêuticas patrocinadas pela indústria com o objetivo de produzir dados para a aprovação de medicamentos pelas autoridades reguladoras. ${ }^{2}$ Em resposta a essas controvérsias, vários interessados diretos preconizaram o registro de ensaios clínicos e o acesso aos dados submetidos às agências reguladoras de medicamentos como ferramentas essenciais de transparência, visando melhorar a tomada de decisões informadas por clínicos, pelas autoridades e pela sociedade. ${ }^{2}$

Apesar do amplo apoio à transparência, sua implementação enfrenta desafios significativos. A transparência dos dados farmacêuticos tem sido dificultada pela qualificação de tais dados como informações sigilosas e por inconsistências no

\footnotetext{
* Tradução oficial para o português realizada pela Organização Pan-Americana da Saúde. Em caso de discrepância, prevalecerá a versão original em inglês.
}

tocante à sobreposição de diferentes esferas reguladoras. Inicialmente, este artigo discutirá os principais desenvolvimentos relacionados ao registro de ensaios clínicos farmacêuticos e ao compartilhamento dos dados enviados às agências reguladoras de medicamentos nas Américas. Em seguida, apresentará os casos de três países, mostrando como implementaram medidas essenciais para a transparência e quais desafios persistem. Finalmente, o artigo sugerirá como a revisão ética de pesquisas, processo presente em todas as jurisdições, pode ser usada para melhorar a transparência de dados.

Os três países selecionados, que representam os quatro principais grupos linguísticos das Américas, contam com fortes indústrias farmacêuticas em diferentes estágios de desenvolvimento: mercado maduro (Canadá), mercado emergente (Brasil) e mercado emergente de segundo nível (Argentina). A maioria dos países latino-americanos se

\section{MENSAGENS-CHAVE}

- As agências reguladoras de saúde nacionais envolvidas na governança de pesquisas relacionadas à saúde devem harmonizar e simplificar tanto a governança dos comitês de ética em pesquisa quanto seus requisitos de transparência, em diferentes regimes de regulação.

- As agências reguladoras de medicamentos dos Estados membros da Organização Pan-Americana da Saúde devem seguir a abordagem adotada pela Agência Europeia de Medicamentos com relação aos dados clínicos usados para a tomada de decisões regulatórias - ou seja, que os dados não constituam segredo comercial e que o ônus recaia sobre os laboratórios farmacêuticos se quiserem argumentar que certos dados específicos devem ser mantidos em sigilo.

- Os comitês de ética em pesquisa podem atuar como coordenadores para promover a adoção de padrões de transparência, na medida em que a estrutura de governança destes comitês reflita seu caráter como entidades de utilidade pública.

Faculdade de Direito e Escola de Saúde Pública Dalla Lana, Universidade de Toronto, Canadá
2 Faculdade de Direito da Universidade de Toronto, Canadá

Este é um artigo de acesso aberto distribuído sob os termos da Licença Creative Commons Attribution-NonCommercial-NoDerivs 3.0 IGO, que permite o uso, distribuição e reprodução em qualquer meio, desde que o trabalho original seja devidamente citado. Não são permitidas modificações ou uso comercial dos artigos. Em qualquer reprodução do artigo, não deve haver nenhuma sugestão de que a OPAS ou o artigo avaliem qualquer organização ou produtos específicos. Não é permitido o uso do logotipo da OPAS. Este aviso deve ser preservado juntamente com o URL original do artigo. 
Quadro 1: Autoridades reguladoras nacionais (ARNs) nas Américas

ARNs reconhecidas pela OPAS como referências regionais em medicamentos e produtos biológicos

- Argentina: Administración Nacional de Medicamentos, Alimentos y Tecnología Médica (ANMAT)

- Brasil: Agência Nacional de Vigilância Sanitária (ANVISA)

- Canadá: Health Canada (HC)

- Chile: Instituto de Salud Pública (ISP)

- Colômbia: Instituto Nacional de Vigilancia de Medicamentos y Alimentos (INVIMA)

- Cuba: Centro para el Control Estatal de Medicamentos, Equipos y Dispositivos Médicos (CECMED)

- Estados Unidos: Food and Drug Administration (FDA)

- México: Comisión Federal para la Protección contra Riesgos Sanitarios (COFEPRIS)

ARNs classificadas como avaliadas ou pré-avaliadas pela OPAS

- Argentina: Administración Nacional de Medicamentos, Alimentos y Tecnología Médica (ANMAT)

- Bahamas: Bahamas National Drug Agency (BNDA)

- Barbados: Barbados Drug Service (BDS)

- Bolívia: Agencia Estatal de Medicamentos y Tecnologías en Salud (antiga Unidad de Medicamentos y Tecnologías en Salud)

- Brasil: Agência Nacional de Vigilância Sanitária (ANVISA) - Ministério da Saúde

- Canadá: Health Canada (HC)

- Chile: Instituto de Salud Pública (ISP)

- Costa Rica: Consejo Nacional de Investigación en Salud (CONIS), CEPs universitários, Caja Costarricense de Seguro Social (CCSS)

- Cuba: Centro para el Control Estatal de Medicamentos, Equipos y Dispositivos Médicos (CECMED)

- Equador: Instituto Nacional de Investigación en Salud Pública (INSPI) (antigo Instituto Nacional de Higiene y Medicina Tropical)

- El Salvador: Dirección Nacional de Medicamentos

- Estados Unidos: Food and Drug Administration (FDA)

- Guatemala: Departamento de Regulación y Control de Productos Farmacéuticos y Afines

- Guiana: Food and Drug Department (FDD)

- Haiti: Direction de la Pharmacie, du Médicament et de la Médicine Traditionnelle (DNM/MT)

- Honduras: Agencia de Regulación Sanitaria (ARSA) (antiga Dirección General de Regulación Sanitaria)

- Jamaica: Standards and Regulation Division (DSR)

- México: Comisión Federal para la Protección contra Riesgos Sanitarios (COFEPRIS)

- Panamá: Dirección Nacional de Farmacia y Drogas

- Paraguai: Dirección Nacional de Vigilancia Sanitaria

- Peru: Dirección General de Medicamentos, Insumos y Drogas (DIGEMID)

- República Dominicana: Dirección General de Medicamentos, Alimentos y Productos Sanitarios (DIGEMAPS)

- Suriname: Autoridade reguladora nacional

- Trinidad e Tobago: Agência reguladora nacional

- Venezuela: Instituto Nacional de Higiene "Rafael Rangel” (IHRR)

Obs.: Todas as agências acima operam como parte dos respectivos ministérios da saúde nacionais. No Caribe anglófono, a Agência de Saúde Pública do Caribe (CARPHA), subordinada à OPAS, participa da definição de normas uniformes. Há também a Rede Pan-Americana de Harmonização da Regulamentação Farmacêutica (PANDRH ou PAHRF) da OPAS.

encontra na última categoria. A Argentina e o Brasil representam países com um número cada vez maior de ensaios clínicos. ${ }^{3}$ As agências reguladoras de medicamentos dos três países são reconhecidas como autoridades regionais de referência (Quadro 1). Considerando-se a diversidade da região em termos de desenvolvimento industrial e análise pelas autoridades reguladoras, esses estudos de caso não visam ser exaustivos nem totalmente representativos. Em vez disso, esclarecem várias iniciativas, resultados e desafios.

\section{AVANÇOS NA TRANSPARÊNCIA EM ENSAIOS CLÍNICOS NAS AMÉRICAS}

Na última década, a OMS, a OPAS, governos nacionais, agências financiadoras, revistas científicas e alguns laboratórios farmacêuticos patrocinadores nas Américas tomaram medidas para promover a transparência. ${ }^{2} \mathrm{O}$ registro de ensaios clínicos, a primeira iniciativa deste tipo a obter amplo apoio, é agora obrigatório em muitos países, apesar de lacunas óbvias (Quadro 2). ${ }^{2}$ A OMS reconhece os registros do Brasil, Cuba e Peru como registros primários na região da OPAS para sua Plataforma Internacional para Registros de Ensaios Clínicos. ${ }^{5}$ Ao mesmo tempo, ocorreu um aumento significativo no registro de ensaios nas Américas. 3,6

Em relação ao compartilhamento de dados farmacêuticos, até agora, nenhuma autoridade reguladora nacional das Américas seguiu o exemplo da Agência Europeia de Medicamentos (EMA), que primeiro facilitou o acesso a tais dados em 2010 e, em 2014, implementou uma 


\begin{tabular}{l} 
Quadro 2: Status dos Estados \\
Membros da OPAS \\
\hline Estados com obrigatoriedade e requi- \\
sitos formais de registro de ensaios \\
clínicos* \\
- Brasil \\
- Colômbia \\
- El Salvador \\
- Estados Unidos \\
- Guatemala \\
- Panamá \\
- Peru \\
- Uruguai \\
Estados com registros nacionaist \\
- Argentina \\
- Brasil \\
- Cuba \\
- Estados Unidos \\
- México \\
- Peru \\
* Compilado com base na pesquisa da OMS \\
sobre perfis farmacêuticos nacionais nas \\
Américas; informações de cada país envia- \\
das com suas políticas atuais verificada nos \\
sites das agências reguladoras nacionais. \\
O site contém apenas países que incluem a \\
obrigatoriedade de registro em sua estrutura \\
regulatória de medicamentos. Alguns países \\
(como o Canadá) têm requisitos de regis- \\
tro apenas parciais, por meio de diretrizes \\
de agências de financiamento que também \\
fazem parte das Boas Práticas Clínicas. \\
+ Os registros da Argentina, México e EUA \\
não são reconhecidos como registros primá- \\
rios pela OMS. \\
\hline
\end{tabular}

política que adota como princípio que todos os dados de ensaios clínicos de fase 1, 2 e 3 utilizados para aprovação regulamentar de medicamentos devem ser disponibilizados ao público. ${ }^{7}$ A política de 2014 parte da premissa de que os dados clínicos não são segredos comerciais, transferindo para as empresas o ônus de mostrar por que o sigilo de dados específicos seria necessário. Nas Américas, nem mesmo os Estados Unidos - cujo Food and Drug Administration Amendments Act (FDAAA) de 2007 inclui obrigações de divulgação - exigem o registro de todos os ensaios clínicos, nem publicam prospectivamente todos os dados de tais ensaios. ${ }^{8}$ As leis nacionais de acesso à informação de vários países fornecem algum nível de acesso aos dados mantidos pelos governos; porém, nesses regimes, as autoridades reguladoras de medicamentos têm amplos poderes discricionários sobre as solicitações de acesso a dados.

\section{BRASIL}

Desde 2010, o Brasil vem implementando políticas relativamente bem-sucedidas de transparência e acesso a dados. O Registro Brasileiro de Ensaios Clínicos (ReBEC) tem como objetivo disponibilizar informações sobre todos os ensaios disponíveis, em inglês, português e espanhol. ${ }^{9} \mathrm{O}$ cadastro no ReBEC é obrigatório para todos os ensaios clínicos que envolvam medicamentos ainda não oficialmente aprovados e envolvam pesquisadores ou participantes brasileiros. A comprovação de cadastro na Plataforma Internacional para Registros de Ensaios Clínicos da OMS (ICTRP) ou em outro registro reconhecido pelo ICMJE é obrigatória para obter a anuência da autoridade reguladora nacional. ${ }^{10}$ De 2010 a 2015, 3112 protocolos foram registrados no ReBEC, ${ }^{11}$ com um aumento no cadastro tanto de ensaios financiados pelo Estado como daqueles patrocinados pela indústria farmacêutica. ${ }^{12}$ Em 2016 e 2017, o número de ensaios registrados foi 1.162 e 1.279 , respectivamente. O ReBEC dá acesso a resumos dos ensaios clínicos, de acordo com o Conjunto de Dados de Registro de Ensaios da OMS. Os relatórios dos ensaios clínicos são submetidos à Agência Nacional de Vigilância Sanitária (ANVISA), mas só podem ser acessados mediante solicitação.

A ANVISA coordena o acesso aos dados conforme a Lei de Acesso à Informação. Qualquer pessoa pode solicitar dados de pesquisa para utilização, reutilização ou redistribuição, desde que cite a autoria e a origem dos dados. A ANVISA criou um repositório de dados aberto, denominado Plano de Dados Abertos, que atende aos padrões de qualidade das normas vigentes. $\mathrm{O}$ acesso a dados pode ser restrito para proteger direitos fundamentais ou o interesse da sociedade e do Estado. ${ }^{13}$ Uma resolução do Conselho Nacional de Saúde exige que os pesquisadores envolvidos em ensaios que tenham recebido financiamento público ou privado publiquem seus resultados. A interrupção de qualquer pesquisa e a não publicação dos resultados devem ser explicadas ao Comitê de Ética em Pesquisa (CEP) e à agência nacional de CEPs, a Comissão Nacional de Ética em Pesquisa (CONEP). ${ }^{14}$

Os avanços na promoção da transparência aparentam estar ameaçados pelas restrições orçamentárias e escassez de pessoal da ReBEC. ${ }^{15} \mathrm{Na}$ esfera regulatória, o compartilhamento de dados pode também ser afetado por acordos de sigilo entre a ANVISA e outras agências reguladoras.

\section{ARGENTINA}

A Argentina elaborou políticas para registro obrigatório de ensaios clínicos e acesso a dados de pesquisa. Porém, tais iniciativas foram implementadas de maneira limitada, em parte impedidas por questões de jurisdição.

A Argentina promulgou o Regime de Boas Práticas Clínicas em Pesquisa Farmacológica (Disposição 6677/10 ANMAT), aplicável a pesquisas que visam obter dados para aprovação regulatória e registro de produtos. As jurisdições locais também podem impor requisitos adicionais. As diretrizes da ANMAT mencionam a Declaração de Helsinque (1964 e versões atuais) e o Conselho de Organizações Internacionais de Ciências Médicas (CIOMS, versão 2002) como referências para sua aplicação, incorporando a Declaração de Helsinque e a versão do CIOMS 2002 como parte das Boas Práticas Clínicas (GCP). Considerando que tanto os patrocinadores quanto os pesquisadores devem respeitar essas diretrizes internacionais, a referência a diferentes diretrizes e diferentes versões em regimes de governança sobrepostos - não exclusivos da Argentina - pode levar a problemas de compatibilidade e uniformidade. As obrigações éticas mais detalhadas e atuais em relação à transparência de pesquisa em saúde preconizadas na versão de 2016 do CIOMS ainda não fazem parte das Boas Práticas Clínicas na Argentina.

A Argentina criou o Registro Nacional de Pesquisa em Saúde (RENIS) para aumentar o registro de ensaios clínicos e outras pesquisas em saúde. ${ }^{16}$ Toda pesquisa financiada pelo Ministério da Saúde ou sujeita aos regulamentos da Administração Nacional de Medicamentos, Alimentos e Tecnologia Médica (ANMAT) deve ser registrada para receber autorização. A implementação de requisitos de registro para outros ensaios clínicos realizados nas províncias depende das autoridades de saúde locais. O RENIS também contém informações sobre conselhos de ética em pesquisa, patrocinadores, pesquisadores e organizações representativas de pesquisa clínica. Em 2016 e 2017, haviam 145 e 180 projetos de pesquisa cadastrados no 
RENIS, ${ }^{17}$ respectivamente, enquanto 191 e 125 ensaios clínicos haviam sido cadastrados no banco de dados da ANMAT para estudos de farmacologia clínica ${ }^{18}$ no mesmo período. As diretrizes da ANMAT contêm regras sobre sigilo do paciente e divulgação de dados (como relatórios de ensaios clínicos) para a ANMAT, mas não para o público.

Diferentes estatutos afetam o acesso a dados de pesquisa. As leis que regem o acesso à informação exigem que agências governamentais, estaduais e descentralizadas forneçam acesso a quaisquer dados sob seu controle. ${ }^{19}$ A Lei do "Habeas Data" também regula o acesso a dados. ${ }^{20}$ Existem outras obrigações de privacidade em relação aos dados pessoais. Porém, se os dados são anonimizados, o acesso não pode ser restrito se esse acesso é para fins científicos ou de interesse público. Dados anonimizados também podem ser compartilhados e transferidos internacionalmente sem necessidade consentimento. $^{21}$ A lei caracteriza os dados de segurança e eficácia submetidos à ANMAT como segredos comerciais ou dados comerciais. ${ }^{22}$ Isso pode criar desafios para a implementação do compartilhamento de dados com pesquisadores independentes e com o público.

A Argentina é um dos países com o maior índice de registro de ensaios clínicos por população, ${ }^{3}$ o que torna o RENIS um esforço valioso. Embora o registro exiba dados resumidos dos ensaios clínicos, o RENIS não cumpre o padrão de dados da OMS, nem fornece acesso a dados em idiomas da OPAS (inglês, português e francês) que não o espanhol. ${ }^{23}$ Isso pode impedir a visibilidade da pesquisa local para atrair ensaios internacionais. ${ }^{9}$ Além disso, o acesso a dados de pesquisa é regulamentado por múltiplas estruturas legais. Isso cria confusão em relação ao direito dos pesquisadores e das organizações da sociedade civil de acessar dados.

\section{CANADÁ}

O Canadá não possui um registro de ensaios próprio e abrangente. O Banco de Dados de Ensaios Clínicos (Clinical Trials Database) da Health Canada fornece algumas informações sobre ensaios clínicos, mas tem como objetivo principal estimular o recrutamento para pesquisas. ${ }^{24} \mathrm{O}$ registro de ensaios e a divulgação pública de resultados são obrigatórios em instituições financiadas pelo governo federal por meio das normas de ética em pesquisa das agências federais, da Tri Council Policy Statement (TCPS2) ${ }^{25}$ e conforme os requisitos específicos de cada agência de financiamento. ${ }^{26}$ Porém, a agência reguladora federal de medicamentos (Health Products and Food Branch, Health Canada) não exige explicitamente o registro de ensaios clínicos de medicamentos e dispositivos médicos. As Diretrizes de Boas Práticas Clínicas da Health Canada ${ }^{27}$ referem-se aos princípios da Declaração de Helsinque como parte da história das boas práticas clínicas, e o CEP do próprio Health Canada segue a declaração TCPS2. Tanto a Declaração de Helsinque quanto a TCPS2 exigem registro de ensaios clínicos e divulgação de resultados. Tais referências indiretas não são juridicamente vinculantes, embora talvez ainda possam ser consideradas como parte das boas práticas clínicas. Os relatórios oficiais há muito ressaltam a necessidade de melhorar a transparência do cadastro regulatório de ensaios clínicos. ${ }^{28}$ Os ensaios tendem a ser registrados no cadastro da FDA nos EUA, e o registro prévio também é exigido pela maioria das revistas médicas canadenses, se não por todas.

A legislação de acesso à informação pode ser usada para solicitar acesso a dados, mas a Health Canada já insistiu que os solicitantes demonstrem como sua necessidade de acesso à informação supera o potencial prejuízo comercial à empresa que detém os dados, o que dificulta a transparência. Uma emenda de 2014 à Lei de Alimentos e Medicamentos criou uma base legal para a divulgação de dados de ensaios clínicos sem o consentimento do patrocinador após a aprovação do medicamento, que poderiam ser usados para implementar regulamentações adicionais sobre o registro de ensaios. ${ }^{29}$ No entanto, a lei refere-se aos dados de ensaios clínicos como segredos comerciais aparentemente minando o conceito dos dados enquanto bem público. A Health Canada atualmente também exige assinatura de um termo de confidencialidade antes de fornecer acesso aos dados, o que atrasa o acesso e posterior compartilhamento de dados. ${ }^{30}$ Porém, um anteprojeto de regulamento ${ }^{31}$ revela uma possível mudança na abordagem regulatória à transparência de dados. Aparentemente inspirado pela abordagem da EMA, o anteprojeto afirma que "resumos clínicos, relatórios e dados de apoio de ensaios clínicos" apresentados durante processo de avaliação de medicamentos não mais serão considerados segredos comerciais após uma decisão regulamentar final. Isso transferiria para os laboratórios farmacêuticos o ônus de demonstrar por que a agência reguladora deve manter dados específicos em sigilo. $\mathrm{O}$ regulamento não se manifesta a respeito da exigência de que os pesquisadores assinem um termo de confidencialidade, uma prática que pode continuar a dificultar o acesso e o compartilhamento de dados. O anteprojeto também não aborda a questão do registro de ensaios, o que exigiria outro novo regulamento.

\section{DESAFIOS À IMPLEMENTAÇÃO E ESTRATÉGIAS REGIONAIS}

Um desafio fundamental a qualquer estratégia coerente de transparência de dados, particularmente no que diz respeito ao acesso a dados de ensaios clínicos sobre produtos farmacêuticos, é o fato de que regimes regulatórios distintos, mas sobrepostos, determinam se, e em qual medida, o compartilhamento de dados pode ocorrer. A maioria dos países possui diretrizes de ética específicas para pesquisas financiadas com recursos públicos. Inspiradas por diretrizes internacionais, como a Declaração de Helsinque ${ }^{32}$ e o CIOMS, citados com frequência, ${ }^{33}$ as diretrizes cada vez incluem mais obrigações de transparência, como registro e divulgação resumida de resultados.

Em países como o Canadá, os ensaios clínicos farmacêuticos financiados pela iniciativa privada porém realizados em instituições financiadas pelo governo federal devem obedecer a essas diretrizes. Os regulamentos sobre alimentos e medicamentos, inclusive os requisitos de boas práticas clínicas, geralmente se referem a diretrizes internacionais de ética. ${ }^{32}$ No entanto, os patrocinadores da indústria insistem - e os regimes regulatórios geralmente aceitam - que os dados de ensaios clínicos sejam segredos comerciais. Isso cria barreiras ao compartilhamento de dados, embora os regimes de acesso à informação geralmente permitam que os pesquisadores solicitem acesso a dados regulatórios após a aprovação do produto. Porém, nestes casos, as agências reguladoras tendem a exercer seu poder discricionário e frequentemente sofrem pressão para respeitar a insistência do setor em manter tais dados sob sigilo.

Os CEPs podem insistir no acesso aos dados como um requisito de ética essencial e garantir que os compromissos de 
transparência sejam adicionados aos termos de consentimento livre e informado apresentados aos sujeitos de pesquisa. Garantir a obtenção de consentimento individual para o compartilhamento de dados também pode ajudar a resolver possíveis preocupações sobre a privacidade das informações pessoais de saúde contidas nos dados de ensaios clínicos. Em todos os países, os ensaios clínicos de produtos farmacêuticos precisam receber parecer favorável do CEP correspondente antes de recrutar seres humanos. É amplamente reconhecido, inclusive nas normas internacionais de boas práticas clínicas, que os CEPs tem a obrigação fundamental de proteger os direitos, a segurança e o bem-estar dos sujeitos de pesquisa. Se como reconhecem as diretrizes nacionais e internacionais de ética - a transparência é um componente essencial da pesquisa ética, os CEPs devem exigir que os pesquisadores e patrocinadores estabeleçam compromissos específicos de transparência como condição para a aprovação ética. Além disso, considerando o reconhecimento cada vez maior do papel dos CEPs após a aprovação do protocolo de pesquisa, não resta dúvida de que esses Conselhos devem verificar ativamente e, na medida do possível, exigir a aplicação das normas de transparência. ${ }^{34}$

Reconhecemos o quanto é difícil conseguir isso. Mesmo na Europa, onde a transparência enfrenta menos barreiras regulatórias, os CEPs carecem de procedimentos adequados para verificar a publicação dos resultados ou minimizar a publicação seletiva. ${ }^{35}$ Nas Américas, as regras de diferentes países sobre transparência em pesquisa também se refletem em diferentes regimes de governança para os CEPs. Alguns países têm uma estrutura administrativa coerente e centralizada, com regras uniformes para todos os CEPs, que podem facilitar o cumprimento das normas de transparência. Por exemplo, no Brasil, todos os CEPs institucionais são responsáveis por analisar os ensaios realizados em sua instituição. No entanto, a CONEP revisa os pareceres dos CEPs e pode solicitar mudanças. A CONEP também autoriza, cadastra e monitora os CEPs institucionais. Embora a estrutura brasileira favoreça a prestação de contas pública e análise coerente dos CEPs, a CONEP sofre com subfinanciamento. ${ }^{9}$

Em outras jurisdições, os CEPs responsáveis por ensaios clínicos patrocinados pela indústria seguem uma estrutura mais orientada para o mercado. Em países como o Canadá (em algumas províncias importantes), os EUA e a Argentina (exceto na Província e Cidade de Buenos Aires), ${ }^{36}$ a maioria dos estudos patrocinados pela indústria são analisados por CEPs particulares. Esses comitês têm um relacionamento direto de cliente e prestador de serviços com os patrocinadores do setor, e competem pela clientela. ${ }^{37}$ São partes interessadas na indústria de produção de conhecimento que apoia os setores de dispositivos médicos e farmacêuticos e opera sob as mesmas normas do mercado - inclusive as normas de sigilo comercial. ${ }^{38}$ A governança dos CEPs alinhadas com o mercado nas pesquisas patrocinadas pela indústria parece inadequada para promover e fazer cumprir o registro de ensaios e o compartilhamento de dados. Questões de jurisdição podem criar barreiras adicionais às tentativas de promover uma estrutura de CEPs publicamente responsável. Isso ocorre, por exemplo, na Argentina e no Canadá. Em cada província da Argentina, os CEPs institucionais e comerciais são coordenados por CEPs centrais. A Resolução 1002/2016 criou o Comitê Consultivo Nacional de Ética em Pesquisa, que colabora com os CEPs provinciais e promove coordenação. Suas atribuições incluem o registro de CEPs que operam em instituições federais ou agências descentralizadas do Ministério da Saúde; embora ainda não tenha jurisdição sobre os CEPs privados. Um estudo de caso argentino recente sobre ensaios patrocinados pela indústria documenta o fracasso do governo e de um CEP privado em impor regras básicas de transparência..$^{39}$ No Canadá, também não há regulamentação ou monitoramento em nível federal dos CEPs. ${ }^{40}$ As províncias mais populosas e que têm a maior atividade de ensaios clínicos de produtos farmacêuticos, Ontário e Quebec, dependem em grande parte de CEPs comerciais privados para análise ética de tais ensaios. Apenas uma província, Terra Nova e Labrador, promulgou leis que explicitamente dão a uma autoridade central de ética em pesquisa a incumbência de organizar a análise de todas as pesquisas realizadas na província por um CEP central. ${ }^{41}$

\section{CONCLUSÃO}

Os países das Américas deram passos importantes rumo à implementação de normas de transparência. Alguns conseguiram progressos significativos na implementação do registro obrigatório de ensaios clínicos e um certo nível de transparência. No entanto, alguns países líderes ainda não conseguiram sequer implementar o registro obrigatório em um cadastro acessível ao público. Embora iniciativas de compartilhamento de dados tenham sido realizadas em vários países, a transparência dos dados de ensaios clínicos de produtos farmacêuticos tem sido prejudicada pela caracterização de tais dados como segredo comercial. Ainda assim, a maioria das jurisdições reconhece o forte embasamento ético da transparência de dados, muitas vezes com referência explícita às diretrizes internacionais de ética em pesquisa. Isso também se reflete nas abordagens ao compartilhamento de dados baseadas nos direitos humanos e no conceito de bem público. ${ }^{38} \mathrm{Se}$ os países da região otimizassem as diferentes esferas regulatórias sobrepostas que regem os dados dos ensaios clínicos e seguissem a liderança da EMA ao adotar o pressuposto de que os dados devem ser acessíveis ao público, eles dariam um passo importante rumo à implementação de uma transparência de dados abrangente.

Para implementar a transparência de dados de saúde nas Américas de maneira coerente, os países precisam conciliar as diferentes regras, diretrizes e ferramentas de governança aplicáveis. Isso significa diferentes agências reguladoras e agências de financiamento sejam responsáveis pela coordenação das regras de transparência aplicáveis em cada nível nacional. O desenvolvimento contínuo de sistemas de CEP publicamente responsáveis, que prestem contas diretamente perante o Estado, deve ser parte da promoção de uma transparência significativa.

Agradecimentos. A Luis Salicrup, Luis Gabriel Cuervo, aos editores do BMJ e aos pareceristas pelo feedback sobre o artigo, e a Suzanne Stephens pela edição. Reconhecemos o apoio financeiro do Programa de Contribuições do Office of the Privacy Commissioner of Canada para um projeto sobre a Acesso a Dados de Ensaios Clínicos de Medicamentos e Privacidade. A Fabio Zicker pela revisão da tradução.

Contribuição dos autores. Ambos os autores declaram que contribuíram de maneira significativa para a concepção e delineamento do trabalho; para a 
aquisição, análise ou interpretação de dados; e para a redação e revisão crítica do artigo. Ambos aprovam a versão final e se responsabilizam por seu conteúdo e por garantir que quaisquer questões relativas à exatidão ou integridade de qualquer parte do trabalho sejam investigadas e resolvidas de maneira apropriada.

Conflito de interesses. Lemos e compreendemos a política do $B M J$ a respeito da declaração de interesses e não temos interesses relevantes a declarar.
Procedência do artigo e revisão por pares. Encomendado; revisão por pares externa.

Declaração. As opiniões externadas neste manuscrito são de responsabilidade do(s) autor(es) e não refletem necessariamente os critérios e políticas da RPSP / PAJPH e/ou da OPAS/OMS.

Série Fortalecimiento da pesquisa em saúde nas Américas. Este artigo faz parte de uma série proposta pela OPAS

\section{REFERÊNCIAS}

1. Organización Panamericana de la Salud/ Organización Mundial de la Salud. Política de Investigación para la Salud. Documento CD49/10, 49. ${ }^{\circ}$ Consejo Directivo, 61. ${ }^{a}$ sesión del Comité Regional de la OMS para las Américas. 2009. http://www1.paho .org/hq/dmdocuments/2009/CD49-10-s .pdf.

2. Krleža-Jeriç K, Lemmens $T$, Reveiz L, Cuervo LG, Bero LA. Prospective registration and results disclosure of clinical trials in the Americas: a roadmap toward transparency. Rev Panam Salud Publica. 2011;30:87-96.

3. Rodríguez-Feria P, Cuervo LG. Progress in trial registration in Latin America and the Caribbean, 2007-2013. Rev Panam Salud Publica. 2017;41:e31.

4. Organización Mundial de la Salud. Pharmaceutical sector country profiles data and reports. Región de las Américas. OPS, 2010. www.who.int/medicines/areas /coordination/coordination_assessment /en/index1.html.

5. Registro Peruano de Ensayos Clínicos. El REPEC ahora es un registro primario de la OMS. 21 Febrero del 2017. www.ensayosclinicos-repec.ins.gob.pe /86-slideshow / 270-el-repec-ahora-es -un-registro-primariode-la-oms.

6. White L, Ortiz Z, Cuervo LG, Reveiz L. Clinical trial regulation in Argentina: overview and analysis of regulatory framework, use of existing tools, and researchers' perspectives to identify potential barriers. Rev Panam Salud Publica. 2011;30:445-52.

7. Agencia Europea de Medicamentos. External guidance on the implementation of the European Medicines Agency Policy on publication of clinical data for medicinal products for human use. 12 de abril del 2017. www.ema.europa.eu/ema /index.jsp?curl=pages / regulation /general/general_content_001799 .jsp\&mid=WC0b01ac0580b2f6ba.

8. Administración de Alimentos y Medicamentos de Estados Unidos. FDA 801 requirements. 2017. https://clinicaltrials .gov/ct2/manage-recs/fdaaa.

9. Freitas CBD, Hossne WS, Dutra S. Progress and challenges of clinical research with new medications in Brazil. En: Homedes
N, Ugalde A, eds. Clinical trials in Latin America: where ethics and business clash. Springer. 2014:151-71.

10. Agencia Nacional de Vigilancia Sanitaria de Brasil. Perguntas e responstas RDC 9/2015: resolução RDC No 9 art.2-3 (2015). 2015. http://portal.anvisa.gov.br /documents/33836/2492465/Perguntas+ e+respostas +sobre+a+RDC $+09+\mathrm{de}+2015$

11. Organización Panamericana de la Salud/ Organización Mundial de la Salud. Relatório de gestão dos termos de cooperação 2015 [Cooperation Terms Management Report 2015]; 2015. www.paho.org/bra /images/relatorio_gestao_2_sem_2015 o16.pdf?ua=1.

12. Silva LR, Laguardia J, Bastos Alves MRA, et al. ReBEC em números: reflexos da política mandatória em pesquisa clínica na trajetória do Registro Brasileiro de Ensaios Clinicos. Cadernos BAD. 2014;2 jul-dez:107-14. https://www.bad. pt/publicacoes/index.php/cadernos /article/view/1187.

13. Agencia Nacional de Vigilancia Sanitaria de Brasil. Sobre a Lei de Acesso a Informação. 2011. http://portal.anvisa.gov.br /sobre-a-lei-de-acesso-a-informcao.

14. Ferreira da Silva C, Ventura M, Castro C. Bioethical perspective of justice in clinical trials. Rev Bioet 2016;24:292-303.

15. Freitas CG, Pesavento TFC, Pedrosa MR, et al. Practical and Conceptual issues of Clinical Trial Registration for Brazilian Researchers. Sao Paulo Med J. 2016;134:28-33.

16. Argentina. Resolución 1480/2011. Apruébase la guía para investigaciones con seres humanos. Bs.As., 13 de septiembre del 2011. http:/ / servicios.infoleg.gob.ar/infoleg Internet/anexos/185000-189999/187206 /norma.htm

17. Argentina, Ministerio de Salud. ReNIS: Registro Nacional de Investigaciones en Salud. www.argentina.gob.ar/salud /registroinvestigaciones.

18. Estudios Clinicos. www.anmat.gov.ar /aplicaciones_net/applications / consultas /ensayos_clinicos/Consulta_EC.asp\#.

19. Ministerio de Justicia y de Derechos Humanos de Argentina. InfoLEG: Información /b14fa962-c1e9-41f5-9524-a290c5b4e98e. e encomendada pelo BMJ, o qual foi responsável pela revisão por pares, edição e decisão de publicar o artigo, sem participação da OPAS. As despesas referentes à publicação desta série em Open Access foram financiadas pela OPAS. Para ler os outros artigos da série em inglês, visite www.bmj.com/health-research -americas; acesso à série em espanhol e português em https://www.paho.org /journal/es/numeros-especiales/fortal ecimiento-investigacion-para-salud -americas
Legislativa. Ley 27275: Derecho de Acceso a la Información Pública, 29 Septiembre 2016. http://servicios.infoleg.gob.ar/infoleg Internet/anexos/265000-269999/265949 /norma.htm.

20. Argentina. Ministerio de Justicia y de Derechos Humanos de Argentina. InfoLEG: Información Legislativa. Ley 25.326: Protección de Datos Personales. 30 Oct 2000. Buenos Aires: InfoLEG; 2016. http:/ / servicios.infoleg.gob.ar/infolegInternet /anexos/60000-64999/64790/texact.htm

21. Outomuro D, Mirabile LM. Confidencialidad y Privacidad en la medicina y en la investigación científica: desde la bioética a la ley. Rev Bioet. 2015;23:238-43. www.scielo.br/pdf/bioet/v23n2/1983 -8034-bioet-23-2-0238.pdf doi:10.1590/1983 $-80422015232062$.

22. Ministerio de Justicia y de Derechos Humanos de Argentina. InfoLEG: Información Legislativa. Ley de confidencialidad sobre información y productos que estén legítimamente bajo control de una persona y se divulgue indebidamente de manera contraria a los usos comerciales honestos: Ley 24-766, 20 dic 1996. Buenos Aires: InfoLEG; 1996. http:// servicios.infoleg.gob.ar/infolegInternet /anexos/40000-44999/41094/norma.htm

23. Organización Panamericana de la Salud/ Organización Mundial de la Salud. Advisory committee on health research: a review of its contributions to health and research for health in the Americas 20092015. 2016. www.paho.org/hq/index.php? option=com_docman\&task=doc_down load\&gid $=36641 \&$ Itemid $=270$.

24. Lemmens T, Gibson S. Decreasing the data deficit: improving post-market surveillance in pharmaceutical regulation. McGill Law J 2014;59:943-88 doi:10.7202/1026134ar.

25. Canadian Institutes of Health Research, Natural Sciences and Engineering Research Council of Canada, and Social Sciences and Humanities Research Council of Canada. Tri-council policy statement: ethical conduct for research involving humans. 9 de diciembre del 2014. www.pre.ethics.gc.ca/pdf/eng /tcps2-2014/TCPS_2_FINAL_Web.pdf.

26. Canadian Institutes of Health Research. Section 2: grants and awards 
management-CIHR's grants and awards guide. 2013. www.cihr-irsc.gc.ca/e/22631 .html\#2-A20.

27. Health Canada. Notice: ICH guideline E6: good clinical practice: consolidated guideline. 2004. www.hc-sc.gc.ca/dhp -mps/alt_formats/hpfb-dgpsa/pdf/prod pharma/e6-eng.pdf.

28. Standing Senate Committee on Social Affairs, Science, and Technology. Canada's clinical trial infrastructure: a prescription for improved access to new medicines. 2012. www.aihealthsolutions.ca/media /Senate-report.pdf.

29. Herder M. The opacity of Bill C-17's transparency amendments. Impact Ethics Blog 23 de junio del 2014. https:/ /impactethics .ca/2014/06/23/the-opacity-of-bill-c-17s -transparency-amendments/.

30. Herder M, Lemmens $T$, Lexchin $J$, et al. Pharmaceutical transparency in Canada: tired of talk. BMJ Blog 6 de junio del 2016. http://blogs.bmj.com/bmj/2016/06/06 /pharmaceutical-transparency-in-canada -tired-of-talk.

31. Gobierno de Canadá, Departamento de Salud. Regulations amending the Food and Drug Regulations (public release of clinical information). 9 de diciembre del 2017. http://gazette.gc.ca/rp-pr/p1 /2017/2017-12-09/html/reg3-eng.html

32. Asociación Médica Mundial. Declaración de Helsinki de la AMM
- principios éticos para las investigaciones médicas en seres humanos. 2013. https:/ / www.wma.net/es/policies-post / declaracion-de-helsinki-de-la-amm -principios-eticos-para-las-investigaciones -medicas-en-seres-humanos /.

33. Consejo de Organizaciones Internacionales de las Ciencias Médicas. Pautas éticas internacionales para la investigación relacionada con la salud con seres humanos. 2016. https://cioms.ch/wp-content / uploads/2017/12/CIOMS-EthicalGuide line_SP_INTERIOR-FINAL.pdf

34. Kolstoe SE, Shanahan DR, Wisely J. Should research ethics committees police reporting bias? BMJ. 2017;356:j1501. PubMed doi:10.1136/bmj.j1501

35. Strech D, Littmann J. The contribution and attitudes of research ethics committees to complete registration and non-selective reporting of clinical trials: a European survey. Res Ethics Rev. 2016;12:123-36 doi:10.1177/1747016115626497.

36. Sabio MF, Bortz JE. Estructura y funcionamiento de los comités de ética en investigación de la Ciudad Autónoma de Buenos Aires y el Gran Buenos Aires. Salud Colect. 2015;11:247-60. http:/ /www scielosp.org/pdf/scol/v11n2/v11n2a08 .pdf. doi:10.18294/sc.2015.687

37. Lemmens $T$, Freedman B. Ethics review for sale? Conflict of interest and commercial research review boards. Milbank
Q. 2000;78:547-84, iii-iv. doi:10.1111 /1468-0009.00185

38. Lemmens T. Pharmaceutical knowledge governance: a human rights perspective. J Law Med Ethics. 2013;41:163-84. doi:10.1111/jlme.12012

39. Homedes N, Ugalde A. The evaluation of complex clinical trial protocols: resources available to research ethics committees and the use of clinical trial registries-a case study. J Med Ethics. 2015;41:464-9. doi:10.1136/medethics-2013-101381

40. Lemmens T. Federal regulation of REB review of clinical trials: a modest but easy step towards an accountable REB review structure in Canada. Health Law Rev. 2005;13:39-50.

41. Newfoundland and Labrador: health research ethics authority act. http:// assembly.nl.ca/Legislation/sr/statutes /h01-2.htm

Como citar (artigo original): BMJ 2018;362:k2493. http:/ /dx.doi.org/10.1136/bmj.k2493.

Manuscrito original em inglês publicado em 16 de julho de 2018 